\title{
Imaging of Domain Wall Inertia in Permalloy Half-Ring Nanowires by Time-Resolved Photoemission Electron Microscopy
}

\author{
J. Rhensius, ${ }^{1,2}$ L. Heyne, ${ }^{1}$ D. Backes,${ }^{1,2}$ S. Krzyk, ${ }^{1}$ L. J. Heyderman, ${ }^{2}$ L. Joly, ${ }^{3}$ F. Nolting, ${ }^{3}$ and M. Kläui ${ }^{1, *}$ \\ ${ }^{1}$ Fachbereich Physik, Universität Konstanz, Universitätsstraße 10, D-78457 Konstanz, Germany \\ ${ }^{2}$ Laboratory for Micro- and Nanotechnology, Paul Scherrer Institut, CH-5232 Villigen PSI, Switzerland \\ ${ }^{3}$ SLS, Paul Scherrer Institut, CH-5232 Villigen PSI, Switzerland
}

(Received 7 April 2009; published 8 February 2010)

\begin{abstract}
Using photoemission electron microscopy, we image the dynamics of a field pulse excited domain wall in a Permalloy nanowire. We find a delay in the onset of the wall motion with respect to the excitation and an oscillatory relaxation of the domain wall back to its equilibrium position, defined by an external magnetic field. The origin of both of these inertia effects is the transfer of energy between energy reservoirs. By imaging the distribution of the exchange energy in the wall spin structure, we determine these reservoirs, which are the basis of the domain wall mass concept.
\end{abstract}

PACS numbers: 75.75.- c, 75.60.Ch, 75.60.Jk

Different concepts have been suggested for next generation solid state memory devices $[1,2]$. These memory concepts are based on domain wall propagation, where the wall velocity governs the device performance. Domain walls were predicted to exhibit an inertialike behavior, which led Döring to introduce the concept of an effective domain wall mass [3]. This is surprising as the magnetization dynamics can be described using the Landau Lifshitz Gilbert (LLG) equation which, in contrast to the classical mechanical second order differential equation, is a first order differential equation that does not contain intrinsic inertia.

The visible consequences for systems that exhibit inertia are the delayed response to an applied generalized force and an oscillatory behavior when relaxing to an equilibrium position. The concept of inertia implies that there is a transfer of energy between different energy reservoirs, as in the transfer of energy between potential and kinetic energies in a mechanical oscillator. In a magnetic system this leads to an effective domain wall mass $[4,6]$. However, the nature of the energy reservoirs in magnetic systems has so far not been identified.

So far, all measurements of domain wall dynamics in wires have been carried out using transport [5-8] or optical techniques $[9,10]$. While these techniques allow for a good time resolution, it is only possible to determine the domain wall positions with no direct determination of the wall spin configuration during the dynamic processes. Various techniques have been used to map the static spin configuration of walls [11-13], without any time resolution. In order to understand where the domain wall mass stems from, direct imaging of the dynamic spin structure with high time resolution as well as a good spatial resolution is necessary.

In this Letter we combine $\mathrm{x}$-ray magnetic circular dichroism photoemission electron microscopy (XMCDPEEM) imaging with a pump-probe technique to image the domain wall spin structure as a function of time. We use nanosecond field pulses to displace a domain wall and de- termine the delay between the field pulse and the onset of the wall displacement as the oscillation frequency of the wall when it relaxes back to its equilibrium position. To determine the origin of this inertia behavior, we investigate the spin structure and find that the wall spin structure deforms during the field pulse, thereby storing exchange energy. We directly image the distribution of the exchange energy in the wall as a function of time and determine the exchange constant to be the governing parameter for the inertia.

We study the domain wall dynamics in half-ring Permalloy nanowires [Fig. 1(a)], where shape anisotropy allows us to tailor the spin structure [11,12]. An image of the spin configuration at rest is shown in Fig. 1(b). We observe an asymmetric transverse domain wall [11] that has been trapped by the applied constant magnetic field $B_{\text {const }}$. The domain wall consists of a central triangular domain (TD) with homogeneous magnetization pointing down and two boundaries, one on the left $\left(B_{L}\right)$ and one on the right $\left(B_{R}\right)$ side of the TD [Fig. 1(b)]. The spins rotate between the triangular domain and the region at the bottom structure edge within an area denoted as $\left(B_{E}\right)$.

The high time resolution of our XMCD-PEEM [14] is achieved by triggering the detector on a single $70 \mathrm{ps}$ wide $\mathrm{x}$-ray pulse with a variable time shift with respect to the field excitation [15]. In order to create a pulsed in-plane magnetic field, a laser pulse with a pulse width of 15 ps and a pulse repetition rate of $63 \mathrm{MHz}$, which is synchronized with the synchrotron, is used to trigger a photodiode, which causes an electrical current to pass through a stripline. The resulting shape [Fig. 3(d) (black curve)] is extracted from the intensity variations on the strip line due to the change of the voltage during the current pulse. A field pulse amplitude of $3.5 \mathrm{mT}$ is reached after a very short rise time of $150 \mathrm{ps}$. The pulse has a width of less than $500 \mathrm{ps}$ and the complete pulse decays after 1800 ps.

To manufacture the samples, first a gold stripline, with a width of $10 \mu \mathrm{m}$ and a thickness of $200 \mathrm{~nm}$, is fabricated by 


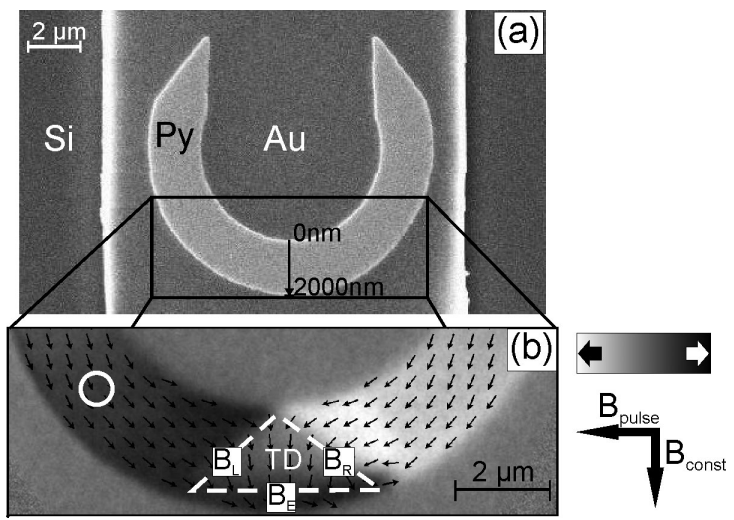

FIG. 1. (a) Scanning electron microscopy image of a Permalloy half-ring nanowire placed on a gold stripline and (b) corresponding XMCD image of an asymmetric domain wall, where the contrast given by the gray-scale bar corresponds to the magnetization direction as visualized by the black arrows.

optical lithography. The half-ring nanowires with a diameter of $6 \mu \mathrm{m}$ and a width of $2 \mu \mathrm{m}$ are fabricated on top of the stripline by electron beam lithography combined with a lift off process [16]. The half-ring material is $20 \mathrm{~nm}$ of Permalloy, deposited in an MBE chamber. To restore the magnetic domain wall back to its equilibrium position, a $4 \mathrm{mT}$ field, $B_{\text {const }}$, is permanently applied using permanent magnets located underneath the sample (for field directions, see Fig. 1).

Images are taken at various moments in time with respect to the field pulse. The field pulse starts at a relative time shift of $11.05 \mathrm{~ns}$ and the shift is increased in steps of 50 ps. In Fig. 2 an image of the wall position just before the beginning of the domain wall motion [Fig. 2(a)] and during the field pulse [Fig. 2(b)] with a time difference of $200 \mathrm{ps}$ is shown. We see that the wall $\left(B_{L}\right)$ is shifted by $440 \mathrm{~nm}$ to the left [compare stars in Figs. 2(a) and 2(b)], yielding a very high velocity of $2200 \mathrm{~m} / \mathrm{s}$ for this part of the wall in line with earlier measurements [9]. Slower average velocities obtained in transport measurements [2] are due to the fact that the velocity is limited at long time scales by the Walker breakdown [17].

In order to analyze the domain wall during the displacement, we extract several spatially averaged line profiles [parallel to the green and red dashed lines in Fig. 2(a)

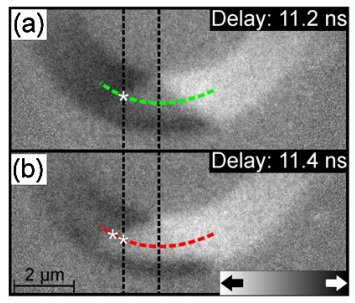

FIG. 2 (color online). Spin configuration in the asymmetric transverse domain wall (a) during the field pulse, just before the domain wall starts to move, corresponding to the time shift $11.2 \mathrm{~ns}$, and (b) $200 \mathrm{ps}$ later where $B_{L}$ is displaced by $440 \mathrm{~nm}$. and 2(b)] across the nanowire [Fig. 3(a)]. The $2000 \mathrm{~nm}$ wide nanowire is divided into 10 spatially averaged slices, each $200 \mathrm{~nm}$ wide. For every slice, line scans are extracted for 55 consecutive time steps separated by $50 \mathrm{ps}$, allowing us to observe the time evolution of the spin configuration in each slice over 2750 ps [see Fig. 3(a)]. Comparing all of the slices, we can see that the time evolution of the magnetization varies from slice-to-slice demonstrating magnetization dynamics that is only revealed by direct spatially resolved imaging. In addition, our experiment compares well with micromagnetic simulations [Fig. 3(b)] [18]. For the simulation, the same nanowire dimensions and field parameters employed in the experiment are used, and standard parameters for Permalloy are chosen: a damping $\alpha=0.02$, a saturation magnetization of $M_{S}=800 \mathrm{kA} / \mathrm{m}$, an exchange constant of $13 \mathrm{pJ} / \mathrm{m}$, a gyromagnetic ratio $\gamma=2.21 \times 10^{5} \mathrm{~m} /$ As, and a lateral cell size of $5 \mathrm{~nm}$. As a guide to the eye, dashed orange lines mark the position of the wall before the pulse and the position of maximum displacement and, since they are not parallel, there is a nonuniform displacement of the left-hand boundary, leading to a change of the spin structure of the domain wall during the displacement.

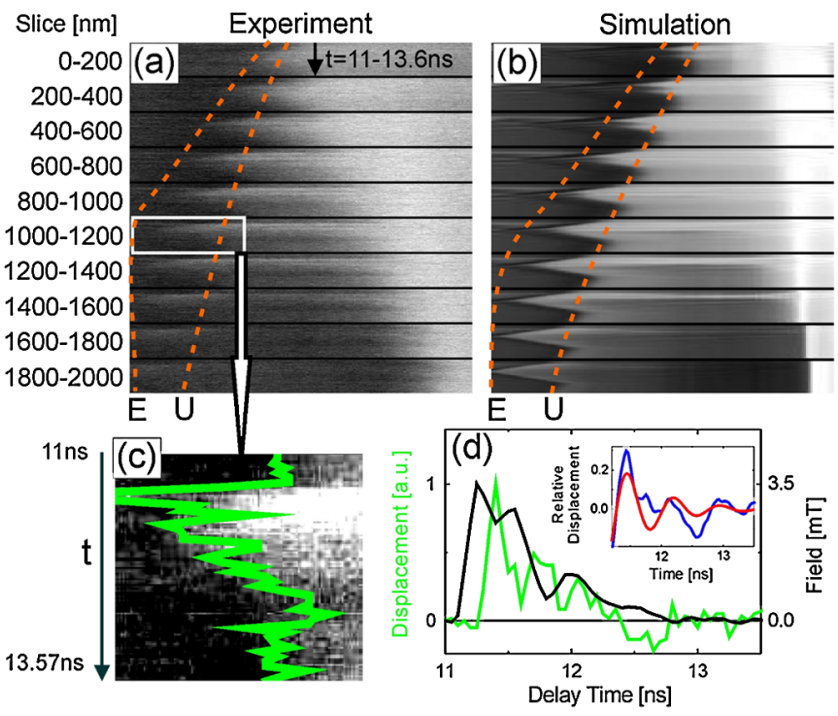

FIG. 3 (color online). Time evolution of the domain wall dynamics (a) in the experiment and (b) in the simulation. The ring shaped structure is divided into ten $200 \mathrm{~nm}$ slices and the average contrast for each slice is given as a function of time for $t=11$ to $13.57 \mathrm{~ns}$. The dashed orange lines indicate the left boundary $\left(B_{L}\right)$ of the wall in the ground state ( $U=$ unexcited) and at the highest wall displacement ( $E=$ excited). (c) Time evolution of $B_{L}$ in the slice 1000-1200 $\mathrm{nm}$. The region corresponds to that indicated by the white frame in Fig. 3(a) and the wall position deduced from the line scans is indicated in green. (d) The domain wall displacement taken from Fig. 3(c) (green) together with the applied field pulse (black). The inset shows the displacement, after subtraction of the running average, yielding the oscillation frequency. The red (or gray) curve is a damped sine fit (red) used to determine the oscillation frequency. 
From the slice 1000-1200 nm in Fig. 3(a), we extract the position of $B_{L}$, as a function of time [shown in green in Fig. 3(c)]. This plot is now shown as a function of time [green line in Fig. 3(d)] together with the time evolution of the field pulse (black line). Here we observe a surprising behavior: First, it is only at 200 ps after the start of the field pulse that the domain wall starts to move in the field direction and reaches its maximum displacement $150 \mathrm{ps}$ after the maximum in the field pulse. Second, as the field pulse decays, the wall moves back due to the permanently applied field, and it undergoes a damped oscillation around its equilibrium position [Fig. 3(d)]. Using a method of subtracting the running average as detailed in Ref. [15] [inset of Fig. 3(d)] we deduce the oscillation frequency to be $1.3 \mathrm{GHz} \pm 0.6 \mathrm{GHz}$.

These two observed phenomena of the delayed displacement and the oscillation are both consequences of what is usually described as inertia. The transfer of energy between different energy reservoirs leads to this inertialike behavior and domain wall mass. In order to identify these energy reservoirs in our magnetic system, we plot the intensity profiles across the wall for the time before the field pulse [11.0 ns, see Fig. 4(a) blue (or dark gray)] and during the field pulse just before the wall starts to move [11.2 ns see Fig. 4(a) green (or gray)]. By comparing the intensity profiles in Fig. 4(a) we see that while the position of $B_{L}$ has not changed in the first $150 \mathrm{ps}$ of the field pulse,
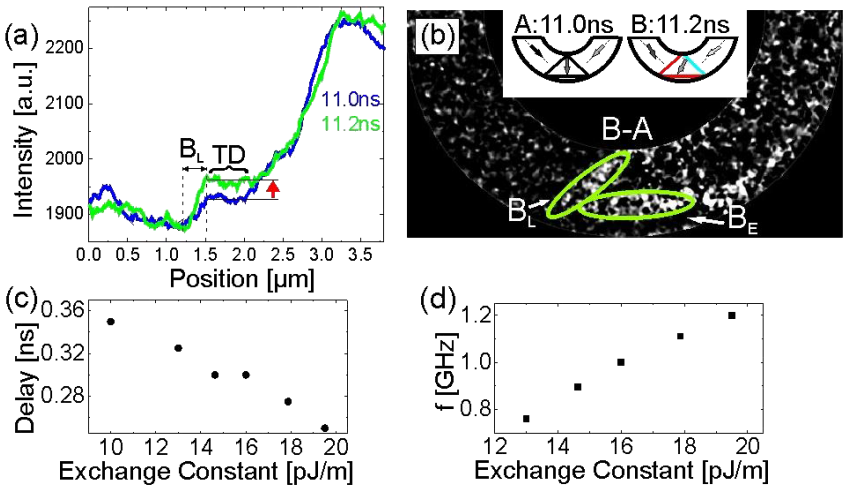

FIG. 4 (color online). (a) Plot of the intensity as a function of position along the dashed line in Fig. 2. The green (11.2 ns) intensity profile corresponds to the green (or gray) dashed curved line in Fig. 2(a). The blue (or dark gray) intensity profile is taken at the same position for the domain wall at rest (11.0 ns). The shift in the intensity level of TD (marked by a red arrow) shows that the spins within TD rotate prior to the displacement. This leads to the local increase in the exchange energy as shown in (b), where the change of the squared gradient of intensity represents a spatial distribution of the exchange energy. The difference between the excited distribution at $11.2 \mathrm{~ns}$ before wall displacement and the distribution of the ground state at $11.0 \mathrm{~ns}$ shows that the exchange energy increases in $B_{L}$ and $B_{E}$ (white areas highlighted by the green frames). The dependence of the delay between the field pulse and the wall displacement (c) and of the oscillation frequency (d) on the exchange constant as deduced from micromagnetic simulations is shown. the angle between the spins to the left and the right of $B_{L}$ is increased from $37^{\circ}$ [blue intensity profile Fig. 4(a)] to $49^{\circ}$ [green intensity profile Fig. 4(a)]. To understand how this change in the profile of $B_{L}$ is related to the delay in the displacement, we have to consider what the effect of the applied field is for the system: as soon as the field pulse is switched on, it generates Zeeman energy, which is then expected to lead to the wall displacement. What we find though is that it first leads to a change in the profile of $B_{L}$, which changes the exchange energy stored in this region. The increased magnetization gradient inside $B_{L}$ [green profile in Fig. 4(a)] means that we have transferred Zeeman energy into exchange energy and thus $B_{L}$ acts as an energy reservoir, which is the prerequisite for this inertialike delay behavior.

To fully analyze this energy transfer, we image the spatial change of exchange energy during the pulse excitation. Since the exchange energy is $E_{\mathrm{ex}} \propto(\nabla \vec{M})^{2}$, the squared gradient of the image intensity represents a relative value for the spatial distribution of the exchange energy inside the domain wall. We show in Fig. 4(b) the difference of the exchange energy for the domain wall prior to the field pulse (11.0 ns) and during the field pulse, just before it starts to move (11.2 ns). Here a clear contrast (white) at the position of $B_{L}$ indicates that the exchange energy increases, so that $B_{L}$ acts as an energy reservoir. Furthermore additional exchange energy is stored in the region $B_{E}$. For both areas, this increase in exchange energy stems from the fact that the spins inside the TD rotate uniformly since they are subjected to the largest torque due to their perpendicular orientation with respect to the field pulse direction. The spins to the left of $B_{L}$ and at the edge below $B_{E}$ do not rotate as much, so that the total angle and thus the gradient across $B_{L}$ and $B_{E}$ increases. This means we can unambiguously attribute the delay to the transfer of the Zeeman energy of the pulse into the exchange energy reservoirs at $B_{L}$ and $B_{E}$.

To further underpin this key finding, we perform micromagnetic simulations to determine the parameter that governs the energy transfer and thus the inertia. Most important is the exchange constant, which governs the efficiency of the transfer from Zeeman to exchange energy. To show the influence on the inertia, we varied the exchange constant and determined the resulting delay and oscillation frequency.

First we find a decreasing delay between the field pulse and the onset of the wall motion with increasing exchange constant as shown in Fig. 4(c). To understand this, we look at the critical wall angle (angle between the spin on both sides of $B_{L}$ and $B_{E}$ ) at the moment that the wall starts moving and we find that this wall angle is smaller for larger exchange constant. The explanation for this behavior is that, in order to trigger the wall motion, a minimum torque is necessary and, for a given angle between any two spins (magnetization gradient across the wall), the torque scales with the exchange constant. So with increasing exchange 
constant this critical torque is already obtained for smaller angles between the spins (smaller magnetization gradients), which is already reached after a shorter delay time.

The second visible consequence of inertia is the behavior of the oscillation frequency of the domain wall, as it relaxes around its equilibrium position. In Fig. 4(d), we find an increase of the oscillation frequency with increasing exchange constant. To analyze this, we look at the formula for the oscillation frequency of a domain wall in a curved wire [1]: We expect: $f \propto \frac{1}{\sqrt{m}}$, with $f$ the oscillation frequency and $m$ the domain wall mass. Since the mass $m$ depends on the domain wall width $\lambda: m \propto \frac{1}{\lambda^{2}}$ [1] and $\lambda=\sqrt{A_{\mathrm{ex}} / K}(K=$ anisotropy, in our case due to shape, $A_{\text {ex }}=$ exchange constant), we expect $f \propto \sqrt{A_{\text {ex }}}$. The dependence of an increasing $f$ with increasing $A_{\text {ex }}$ agrees with that of our simulations [Fig. 4(d)]. So, both the delay and the oscillation frequency strongly depend on the exchange constant, which is thus the governing parameter for the inertia.

To obtain a quantitative result of the domain wall mass, we determine the oscillation frequency $f$ to be $1.3 \mathrm{GHz} \pm$ $0.6 \mathrm{GHz}$. We can deduce with $f^{2}=\frac{Q_{m} H}{4 \pi^{2} m r}$ an effective wall mass for the domain wall of $1.3 \pm 0.1 \times 10^{-24} \mathrm{~kg}$ [4]. The applied field is $H=4 \mathrm{mT}, r=3 \mu \mathrm{m}$ is the radius of the structure and $Q_{m}=2 \mu_{0} M_{s} S$ is the magnetic charge in zero field with the saturation magnetization $M_{S}=$ $860 \mathrm{kA} / \mathrm{m}$ and the cross-sectional area $S=2 \times 10^{-6} \times$ $20 \times 10^{-9} \mathrm{~m}^{2}$. This domain wall mass is in line with previously measured values $[4,6]$.

To check that our observed inertia behavior indeed is related to the inhomogeneous wall spin structure and not an artifact of the measurement, we analyze the spin dynamics away from the wall in the region indicated by the circle in Fig. 1(b). We find that the spins follow the field almost instantaneously as expected from the LLG equation for a macrospin. The frequency of this oscillation is $3.4 \mathrm{GHz} \pm$ $0.6 \mathrm{GHz}$, which corresponds to a precession of the spins around the effective field. From the micromagnetic simulations, we determined the strength of the effective field to be $114 \mathrm{kA} / \mathrm{m}$ and from the Kittel formula [19] an expected precession frequency of $4 \mathrm{GHz}$ is obtained, which agrees very well with the experimentally observed value.

In conclusion, we find that the domain wall inertia stems from the energy transfer between two energy reservoirs, the exchange energy inside the domain wall spin structure, and the Zeeman energy of the wall in the magnetic field. As the field pulse is applied, prior to the onset of motion, the domain wall boundary becomes distorted so storing exchange energy, which we directly image. This leads to a delay between the field pulse excitation and the first wall motion and a damped oscillation, as the wall relaxes to its original position. Micromagnetic simulations indicate that the inertia and the resulting domain wall mass is governed by the exchange parameter.
The authors would like to acknowledge the support of Anja Weber, Michael Horrisberger, Eugen Deckardt, and Christian David with sample fabrication. This work was supported financially by the German Science Foundation (DFG SFB 767, KL 1811), the ERC (Starting Independent Researcher Grant No. ERC 2007-Stg 208162), the EU (RTN Spinswitch, MRTN CT-2006-035327), and the Samsung Advanced Institute of Technology. Some of this work was performed at the Swiss Light Source, Paul Scherrer Institut, Villigen, Switzerland.

*Author to whom correspondence should be addressed. Mathias.Klaeui@uni-konstanz.de Also at Zukunftskolleg, Universität Konstanz, 78457 Konstanz, Germany.

[1] R. Cowburn, U.S. Patent No. WO/2007/132174.

[2] S. S. P. Parkin, M Hayashi, and L. Thomas, Science 320, 190 (2008).

[3] W. Döring, Z. Naturforsch. 3, 373 (1948).

[4] E. Saitoh, H. Miyajima, T. Yamaoka, and G. Tatara, Nature (London) 432, 203 (2004).

[5] M. Hayashi, L. Thomas, C. Rettner, R. Moriya, and S. S. P. Parkin, Nature Phys. 3, 21 (2007).

[6] D. Bedau, M. Kläui, S. Krzyk, U. Rüdiger, G. Faini, and L. Vila, Phys. Rev. Lett. 99, 146601 (2007).

[7] M. Hayashi, L. Thomas, R. Moriya, C. Rettner, and S. S. P. Parkin, Science 320, 209 (2008).

[8] T. Ono, H. Miyajima, K. Shigeto, K. Mibu, N. Hosoito, and T. Shinjo, Science 284, 468 (1999).

[9] D. Atkinson, D. A. Allwood, G. Xiong, M. D. Cooke, C. C. Faulkner, and R. P. Cowburn, Nature Mater. 2, 85 (2003).

[10] G. S. D. Beach, C. Nistor, C. Knutson, M. Tsoi, and J. L. Erskine, NATO Adv. Study Inst. Ser. C, Math. Phys. Sci. 4, 741 (2005).

[11] D. Backes, C. Schieback, M. Kläui, F. Junginger, H. Ehrke, P. Nielaba, U. Rüdiger, L. J. Heyderman, C. S. Chen, T. Kasama, R. E. Dunin-Borkowski, C. A. F. Vaz, and J. A. C. Bland, Appl. Phys. Lett. 91, 112502 (2007).

[12] M. Kläui, C. A. F. Vaz, and J. A. C. Bland, Appl. Phys. Lett. 85, 5637 (2004).

[13] A. Yamaguchi, T. Ono, S. Nasu, K. Miyake, K. Mibuand, and T. Shinjo, Phys. Rev. Lett. 92, 077205 (2004).

[14] J. Stöhr, Y. Wu, B. D. Hermsmeier, M. G. Samant, G. R. Harp, S. Koranda, D. Dunham, and B. P. Tonner, Science 259, 658 (1993).

[15] J. Raabe, C. Quitmann, C. H. Back, F. Nolting, S. Johnson, and C. Buehler, Phys. Rev. Lett. 94, 217204 (2005).

[16] L. J. Heyderman, M. Kläui, B. Nöhammer, C. A. F. Vaz, J. A. C. Bland, and C. David, Microelectron. Eng. 73-74, 780 (2004).

[17] A. Thiaville, Y. Nakatani et al., Spin Dynamics in Confined Magnetic Structures III, edited by B. Hillebrands (Springer, New York, 2003).

[18] http://math.nist.gov/oommf/.

[19] C. Kittel, Phys. Rev. 80, 918 (1950). 